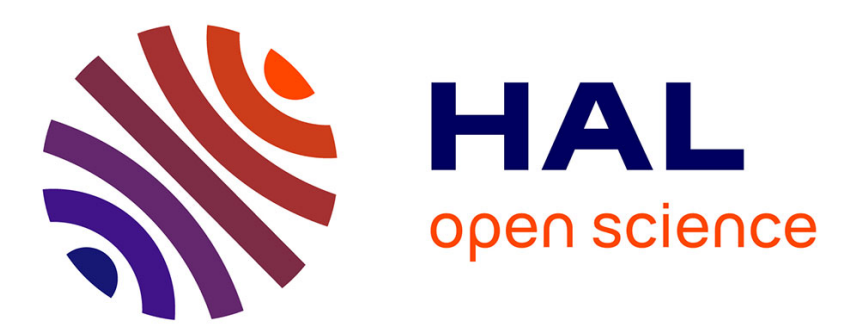

\title{
HST/GHRS Observations of the Velocity Structure of Interplanetary Hydrogen
}

\author{
John T. Clarke, Rosine Lallement, Jean-Loup Bertaux, Hans Fahr, Eric
}

Quémerais, Horst Scherer

\section{- To cite this version:}

John T. Clarke, Rosine Lallement, Jean-Loup Bertaux, Hans Fahr, Eric Quémerais, et al.. HST/GHRS Observations of the Velocity Structure of Interplanetary Hydrogen. The Astrophysical Journal, 1998, 499 (1), pp.482 - 488 . insu-02902256

\section{HAL Id: insu-02902256 https://hal-insu.archives-ouvertes.fr/insu-02902256}

Submitted on 18 Jul 2020

HAL is a multi-disciplinary open access archive for the deposit and dissemination of scientific research documents, whether they are published or not. The documents may come from teaching and research institutions in France or abroad, or from public or private research centers.
L'archive ouverte pluridisciplinaire HAL, est destinée au dépôt et à la diffusion de documents scientifiques de niveau recherche, publiés ou non, émanant des établissements d'enseignement et de recherche français ou étrangers, des laboratoires publics ou privés. 
The Astrophysical Journal, 499:482-488, 1998 May 20

(C) 1998. The American Astronomical Society. All rights reserved. Printed in U.S.A.

\title{
HST/GHRS OBSERVATIONS OF THE VELOCITY STRUCTURE OF INTERPLANETARY HYDROGEN John T. Clarke, ${ }^{1}$ Rosine Lallement, ${ }^{2}$ JeAn-Loup Bertaux, ${ }^{2}$ Hans Fahr, ${ }^{3}$ Eric Quemerais, ${ }^{2}$ ANd Horst SChERER ${ }^{3}$ Received 1997 September 5 ; accepted 1998 January 5
}

\begin{abstract}
We present high-resolution spectra of the emission-line profile of inflowing interplanetary hydrogen atoms along lines of sight with the Earth orbital motion upwind (into the flow), downwind, and across the flow to Doppler-shift the line from the geocoronal emission. The line-center positions, in comparison with hot-model profiles, confirm that the inflow speed of $\mathrm{H}$ atoms far from the Sun $(\sim 50 \mathrm{AU})$ is in the range 18-21 km s$~^{-1}$, which implies a decrease in the velocity distribution of $5-8 \mathrm{~km} \mathrm{~s}^{-1}$ for hydrogen within the solar system, relative to the He flow and to the local interstellar medium. Best-fit values are derived for the speed and effective solar gravity along the three lines of sight by comparison with model profiles convolved with the instrument line-spread function. For the assumed inflow direction, the crossflow line profile requires that the $\mu$-value be slightly less than unity near solar minimum, and a technique is presented for determining the exact inflow direction and $\mu$-value independently of the other parameters. The line widths indicate a broadening along the flow direction in addition to the dynamical effects near the Sun expected from two different hot models, whereas the cross-flow line width is similar to the hot-model profiles. The altered velocity distribution in the inflow direction appears likely to be related to the crossing of the interstellar/interplanetary medium interface structure, although questions remain about the cumulative effects of changing solar activity on the timescale of the $\mathrm{H}$ atom flow through the solar system.

Subject heading: interplanetary medium
\end{abstract}

\section{INTRODUCTION}

The interaction of the local interstellar medium (ISM) with the solar wind can be considered to demarcate the outer boundary of the solar system. The magnetohydrodynamic interaction of the solar wind plasma with the ionized component of the ISM leads to the formation of a so-called interface region, within which both plasma flows are deflected from their original directions. The heliopause as a tangential discontinuity separates the two plasma flows from each other. While they do experience charge-exchange reactions with solar wind protons, the neutral, local interstellar medium (LISM) hydrogen atoms can penetrate through this region and flow into the solar system, forming a wind of interplanetary hydrogen (IPH) atoms. Inflowing ISM $\mathrm{H}$ and $\mathrm{He}$ atoms resonantly scatter the bright solar emission lines $\mathrm{H}_{\mathrm{I}} \lambda 1216$ and $\mathrm{He} \mathrm{I} \lambda 584$, first observed as diffuse Ly $\alpha$ emission, in addition to the geocoronal emission (Bertaux \& Blamont 1971; Thomas \& Krassa 1971). All-sky maps of these emissions have been obtained by the Pioneer, Voyager, and Galileo UV instruments (Pryor et al. 1992; Hall et al. 1993; Ajello et al. 1994). The IPH temperature was determined from fits to absorption cell measurements to be $8000 \mathrm{~K}$ (Bertaux et al. 1976; Wu \& Judge 1980; Bertaux et al. 1985) and the inflow speed to be $20 \mathrm{kms}^{-1}$ (Bertaux et al. 1985). The "inflow" speeds here refer to the region, taken to be at a reference distance of $50 \mathrm{AU}$, before any significant modification of the flow occurs from nearsolar effects. Absorption cell observations are limited to roughly half the sky near the crosswind direction by their limited spectral range, however. The velocity of the $\mathrm{H}$ inflow has also been directly measured by Copernicus

\footnotetext{
${ }^{1}$ Space Physics Research Laboratory, University of Michigan, Ann Arbor, MI 48109-2143.

${ }^{2}$ Service d'Aeronomie du CNRS, BP3, Verrières-le-Buisson 91371, France.

${ }^{3}$ Universität Bonn, Institüt für Astrophysik, Auf dem Hügel 71, D-53121 Bonn, Germany.
}

(Adams \& Frisch 1977) and the International Ultraviolet Explorer (Clarke et al. 1984) at high spectral resolution by observing when the Earth orbital motion is "upwind " into the flow, providing a combined velocity difference of up to $50 \mathrm{kms}^{-1}$ or a $0.20 \AA$ Doppler shift of the IPH H Ly $\alpha$ line from the geocoronal emission. At sufficiently high spectral resolution, both the velocity and line shape can be determined, providing a direct measurement of the velocity dispersion of the inflowing IPH atoms along the line of sight.

The interactions of the IPH with solar gravity, ionization, and radiation pressure have been modeled for comparison with the observational data. The most important processes are (1) charge exchange with solar wind protons and photoionization, which reduces the density of the IPH near the Sun, (2) radiation pressure from the solar UV resonance lines, and (3) solar gravity. The "optically thin" solar radiation pressure at a specific frequency and solar gravity are both $R^{-2}$ forces, and the combined effective potential (described by $\mu=$ solar radiation pressure/solar gravity) leads to a net focusing or divergence in the downwind direction from the Sun. The temperature of the IPH gas also affects the degree of focusing in the downwind direction, in the sense that colder gas is more readily focused, and photoionization and charge exchange with solar wind protons near the Sun (the lifetime to destruction for the $\mathrm{H}$ atom at 1 $\mathrm{AU}=T_{D}$ ) act to decrease the local $\mathrm{H}$ density. The "hot" models for the $\mathrm{H}$ flow take into account these near-solar effects, which change the number density and velocity distribution near the Sun. Initial reports of the Hubble Space Telescope (HST) Goddard High Resolution Spectrograph (GHRS) echelle A spectra (Lallement, Bertaux, \& Clarke 1993; Clarke et al. 1995) have shown that the measured downwind $\mathrm{H}$ velocity is consistent with an inflow of 20 $\mathrm{kms}^{-1}$, from comparison with a hot-model profile. This suggested that the $\mathrm{H}$ flow is decelerated in the solar system with respect to the LISM flow (Lallement \& Bertin 1992; Linsky et al. 1993) and also with respect to the He flow at 26 
$\mathrm{kms}^{-1}$ inside the solar system (Witte et al. 1993). It was proposed that this may occur through charge exchange in the heliospheric interface region, a process which has been modeled by several groups (Ripken \& Fahr 1983; Fahr \& Ripken 1984; Baranov, Lebedev, \& Malama 1991; Malama 1991; Osterbart \& Fahr 1992). However, these observations were in the downwind direction, in which there is substantial modification of the flow by near-solar effects, and the derived velocity is thus model dependent. While it was well known that upwind observations would provide a less model-dependent measurement of the inflow speed, these observations had to wait for the repair of side 1 of the GHRS in the 1993 December HST servicing mission.

In this paper we present $H S T$ observations with the GHRS and echelle A grating in the upwind, downwind, and cross-flow directions, obtained over 1994-1996 for comparison with the earlier spectra and derivation of the properties of the IPH flow within the solar system. We also present a comparison of the observed IPH profiles with the results of two "hot" models (hereafter the RL [Lallement, Bertaux, \& Dalaudier 1985; Quemerais, Lallement, \& Bertaux 1993] and HS [Scherer \& Fahr 1996; Scherer, Fahr, \& Clarke 1997] models), each treating the modifications to the flow near the Sun and neither including any modifications at the heliospheric interface for the results presented in this paper. By this approach we intend to identify any effects that can be attributed to near-solar flow modification and thereby isolate those effects most likely to be due to modifications at the heliospheric interface. The nature and location of the heliospheric interface are fundamental questions in space plasma physics, and the IPH flow of neutral $\mathrm{H}$ atoms carrying the imprint of this interface offers a method of remotely sensing the nature of this interface from the vicinity of the Earth.

\section{OBSERVATIONS AND DATA REDUCTION}

Observations of the IPH H Ly $\alpha$ emission with the HST and GHRS began in 1994 spring and concluded successfully in 1996 spring, with repeated upwind and downwind observations because of incomplete initial observations. All spectra were obtained with the 1".74 square large science aperture (LSA) and echelle A grating with the side 1 CsI Digicon detector. A detailed analysis of the properties of the GHRS in this mode have been presented by Clarke et al. (1995), along with initial observations in the downwind direction. These properties include the measured linespread function (LSF) and a simulation of the instrument response, the measured-grating scatter profile, including any grating ghosts, and the sensitivity of the instrument to diffuse emission. Unless otherwise stated, the spectra presented here have been reduced by the same procedures as in Clarke et al. (1995). The LSF for diffuse emission filling the aperture is nearly rectangular, with a spectral resolution of $0.07 \AA\left(17 \mathrm{kms}^{-1}\right)$. Since the addition of the Corrective Optics Space Telescope Axial Replacement (COSTAR) in 1993 December, there have been two added reflections and a slightly smaller aperture field of view, decreasing the sensitivity to diffuse emission at $1216 \AA$ by roughly a factor of 2 . Since the GHRS instrument is unchanged, the LSF and other characteristics are unchanged. The zero point of the wavelength scale is derived from the observed centroid of the geocoronal Ly $\alpha$ emission after correction for the HST orbital motion, and the dispersion and LSF are well characterized and stable. The main uncertainty in deriving velocities from these spectra is the limited signal-to-noise ratio in the spectra. Pointing locations have been selected for the directions upwind, downwind, and across (perpendicular to) the flow, with the upwind direction as determined from the He flow (Weller \& Meier 1974; Witte et al. 1993). Specific pointings have been toward nearby ISM dust clouds to minimize the chances of contaminating stellar emission in the aperture.

The observing times and directions are listed in Table 1 and plotted in Figure 1, and the observed spectra are plotted in Figure 2. The geocoronal emission is much brighter than the IPH emission and must be fitted and subtracted to reveal the true profile of the IPH emission. For each observation we have convolved a Voigt profile with the GHRS echelle A LSF for diffuse emission filling the aperture, varying independently the assumed temperature and intensity of this convolved profile to obtain the best fit by the method of least squares. In this case, the best-fit temperature represents an effective fit to the actual geocoronal temperature and optical depth along the line of sight, and we have found that this gives a good fit to the observed geocoronal line shape, since the echelle A LSF is much broader than the intrinsic geocoronal line width. We have limited the wavelength range of the fit to avoid any substantial contribution from the IPH emission. While there is no need to vary the central wavelength of the geocoronal line, which appears at rest wavelength, both the line width and central wavelength must be determined for the IPH emissions. To initially fit the IPH emission profile, we have also convolved a Voigt profile with the echelle A LSF and varied independently the central wavelength and intensity of the model, using $20,000 \mathrm{~K}$ for upwind and downwind spectra and 10,000 K for the crosswind spectrum. We have then varied the temperature and intensity of the model at

TABLE 1

HST/GHRS OBSERVATIONS OF THE INTERPLANETARY MEDIUM

\begin{tabular}{|c|c|c|c|c|c|}
\hline Date & $\begin{array}{l}\text { Time } \\
\text { (UT) }\end{array}$ & Pointing & $\begin{array}{l}\text { Exposure Time } \\
\text { (minutes) }\end{array}$ & $\begin{array}{l}\text { Earth LOS Velocity } \\
\qquad\left(\mathrm{km} \mathrm{s}^{-1}\right)\end{array}$ & $\begin{array}{l}\text { Brightness } \\
\text { (R) }\end{array}$ \\
\hline 1994 Apr 7 ........ & $8-10$ & Upwind & 24.9 & +25.4 & 960 \\
\hline 1994 Jun $4 \ldots$ & $5-12$ & Crosswind & 72.5 & -29.8 & 900 \\
\hline 1995 Mar 6-7 ...... & $21-4$ & Downwind & 54.4 & -29.9 & 280 \\
\hline 1995 Mar $26 \ldots \ldots$. & $17-21$ & Upwind & 63.5 & +28.2 & 850 \\
\hline 1996 Mar 9 ....... & $9-14$ & Downwind & 72.5 & -29.8 & 260 \\
\hline
\end{tabular}

NoTE.-All observations used GHRS echelle A and the large science aperture (1".74 square) over 1212.3$1218.7 \AA$. Pointings in (right ascension, declination), epoch 2000 , are upwind $=(252.71,-15.43$ ), downwind $=(71: 85,+17.17)$, and crosswind $=(164: 89,+0.34)$.

${ }^{a}$ Positive line-of-sight (LOS) velocities are approaching, negative are receding. 

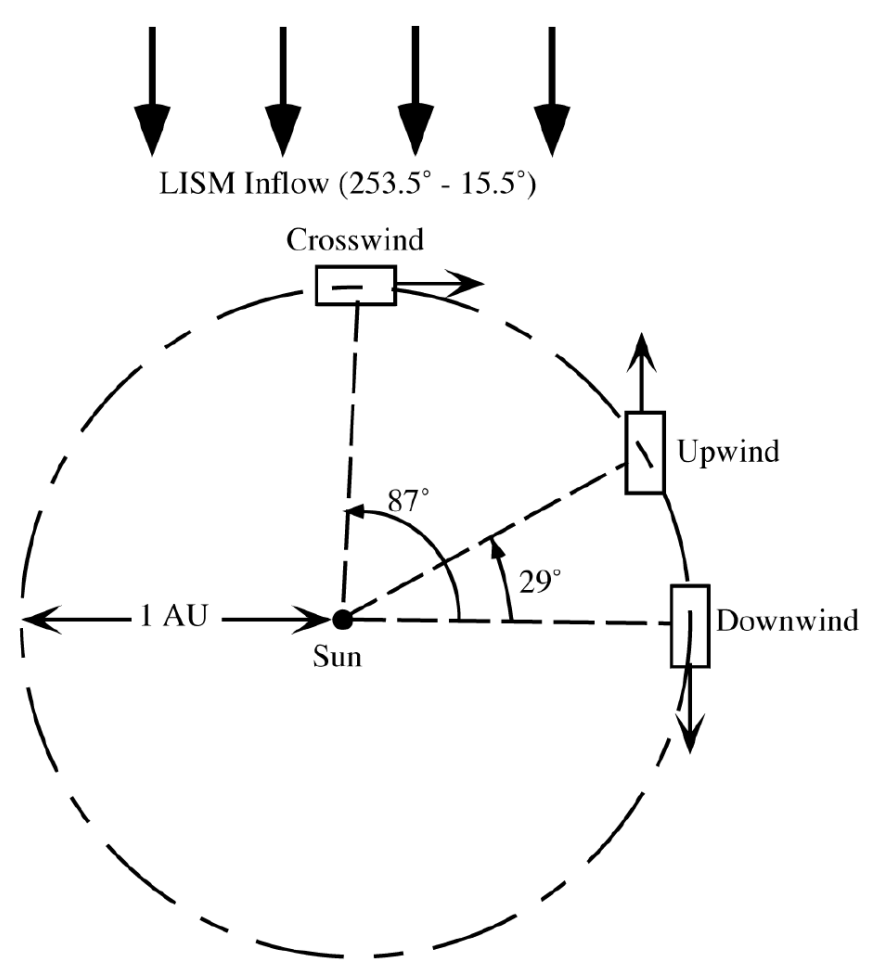

FIG. 1.-Schematic drawing showing orbital orientation of the Earth at the times of $H S T /$ GHRS observations over 1994-1996, looking down from celestial north. Line-of-sight velocity components are indicated and discussed in the text. The observing geometry in 1991 May was presented in Clarke et al. (1995).

the best-fit central wavelength to optimize the fit, and the best-fit values are listed in Table 2 . The model temperature is an upper limit to the actual temperature of the IPH, since the observed line is broadened also in part by the velocity dispersion along the line of sight and the optical depth effects. More detailed modeling must be performed to determine the true local temperature of the inflowing IPH atoms.

The upwind IPH line profiles observed in 1994 and 1995 are qualitatively similar, with a longer integration time and corresponding higher signal-to-noise ratio in the $1995 \mathrm{spec}-$ trum. In comparison with the convolved Voigt profiles, it appears that there is some wavelength structure in the IPH line, particularly in the 1995 spectrum. Higher signal-tonoise data will be needed to confirm this, but this structure may reflect regions of varying flow speed in an inhomogeneous, inflowing IPH. The crosswind spectrum in Figure 2 was obtained pointing opposite to the Earth orbital motion in 1994 and shows some overlap of the geocoronal and redshifted IPH emissions, since these are separated only by the Earth orbital motion of $30 \mathrm{~km} \mathrm{~s}^{-1}$. After subtraction of the geocoronal emission, the blue side of the line in the difference spectrum has an added uncertainty from the potential error in subtraction. The downwind IPH emissions are the faintest and have lower signal to noise, consistent with earlier observations.

\section{COMPARISON OF OBSERVED LINE PROFILES WITH MODEL PROFILES}

\subsection{Upwind}

The upwind IPH line profile provides the most direct measurement of the IPH velocity, with the largest Doppler shift from the geocoronal line, the brightest emission, and the least influence of near-solar effects. The upwind profile has a moderate sensitivity to values of $\mu$, and only a weak sensitivity to values of $T_{D}$ and temperature. From the crosswind spectrum modeling (where the value of $\mu$ is better defined), we adopt values for $\mu=0.8-1.0$, and we consider values of $T_{D}=(1.0-2.0) \times 10^{6} \mathrm{~s}$. Changes in $T_{D}$ produce a small shift of the center of the IPH line in the upwind spectrum, too small to significantly distinguish between different reasonable values of $T_{D}$ (Puyoo, BenJaffel, \& Emerich 1997). Variations in $\mu$ from 0.8 to 1.0 produce a $1-2 \mathrm{kms}^{-1}$ change in the upwind profile centroid, which is large compared with the observed variations from changes in the value of $T_{D}$. Changes in the temperature change the observed width of the line but not the central wavelength. An assumed temperature of $8000 \mathrm{~K}$ gives a line significantly narrower than the observed profile in all cases, and temperatures of the order of 20,000 K are needed to give satisfactory fits. An alternative would be to include a fractional superthermal population in the flow, which would also broaden the line. Lacking further knowledge of such an effect, we have not applied any specific model for a superthermal population at this time. We have varied the model velocity to obtain a best least-squares fit to the observed profile, with the model intensity allowed to vary to minimize $\chi^{2}$ for each assumed velocity. The best-fit line-of-sight velocities far from the Sun are listed in Table 2. The main uncertainties are from the limited signal to noise in the line profile and a $1-2 \mathrm{kms}^{-1}$ difference between the two models used (RL and HS). One caveat is that while the hot models take into account the acceleration of the flow near the Sun for values of $\mu<1$, appropriate to these observations near solar minimum, they

TABLE 2

INTERPLANETARY EMISSION-LINE FITS

\begin{tabular}{llccccc}
\hline \hline \multicolumn{1}{c}{ Date } & Pointing & $\begin{array}{l}\text { Geocoronal } \\
\text { Temperature }^{\mathrm{a}}\end{array}$ & IPH Temperature & $\begin{array}{c}\lambda_{\text {IPH }}{ }^{\mathrm{b}} \\
(\AA)\end{array}$ & $\begin{array}{c}\text { IPH Inflow Speed }^{\mathrm{c}} \\
\left(\mathrm{km} \mathrm{s}^{-1}\right)\end{array}$ & ${\text { IPH } \mu^{\mathrm{d}}}^{-12}$ \\
\hline 1994 Apr 7 & Upwind & 2100 & $25 \pm 10 \times 10^{3}$ & $1215.483 \pm 0.003$ & $18 \pm 2$ & 0.98 \\
1994 Jun 4 & Crosswind & 1800 & $9 \pm 2 \times 10^{3}$ & $1215.781 \pm 0.002$ & $18 \pm 2$ & 1.04 \\
1995 Mar 6-7 & Downwind & 1900 & $30 \pm 15 \times 10^{3}$ & $1215.872 \pm 0.007$ & $20 \pm 4$ & 1.01 \\
1995 Mar 26 & Upwind & 2400 & $17 \pm 4 \times 10^{3}$ & $1215.459 \pm 0.002$ & $21 \pm 2$ & 1.08 \\
1996 Mar 9 & Downwind & 3100 & $15 \pm 5 \times 10^{3}$ & $1215.893 \pm 0.004$ & $15 \pm 3$ & 0.93 \\
\hline
\end{tabular}

a Temperatures (in kelvins) are determined by least-squares fit to a Voigt profile convolved with the echelle A line-spread function and are thereby only representative of the actual velocity distribution and optical depth.

${ }^{b}$ Best-fit IPH line-center wavelength.

${ }^{\mathrm{c}}$ Best-fit inflow speed from comparison with hot-model profiles, assuming $\mu=0.99, T_{D}=1.25 \times 10^{6}$, and best-fit IPH temperature.

${ }^{\mathrm{d}}$ Calculated values of $\mu$ for the IPH line-of-sight motion in the solar rest frame, using solar Ly $\alpha$ fluxes from the SOLSTICE experiment (Tobiska et al. 1997) and the solar line profile from LeMaire et al. (1978). 

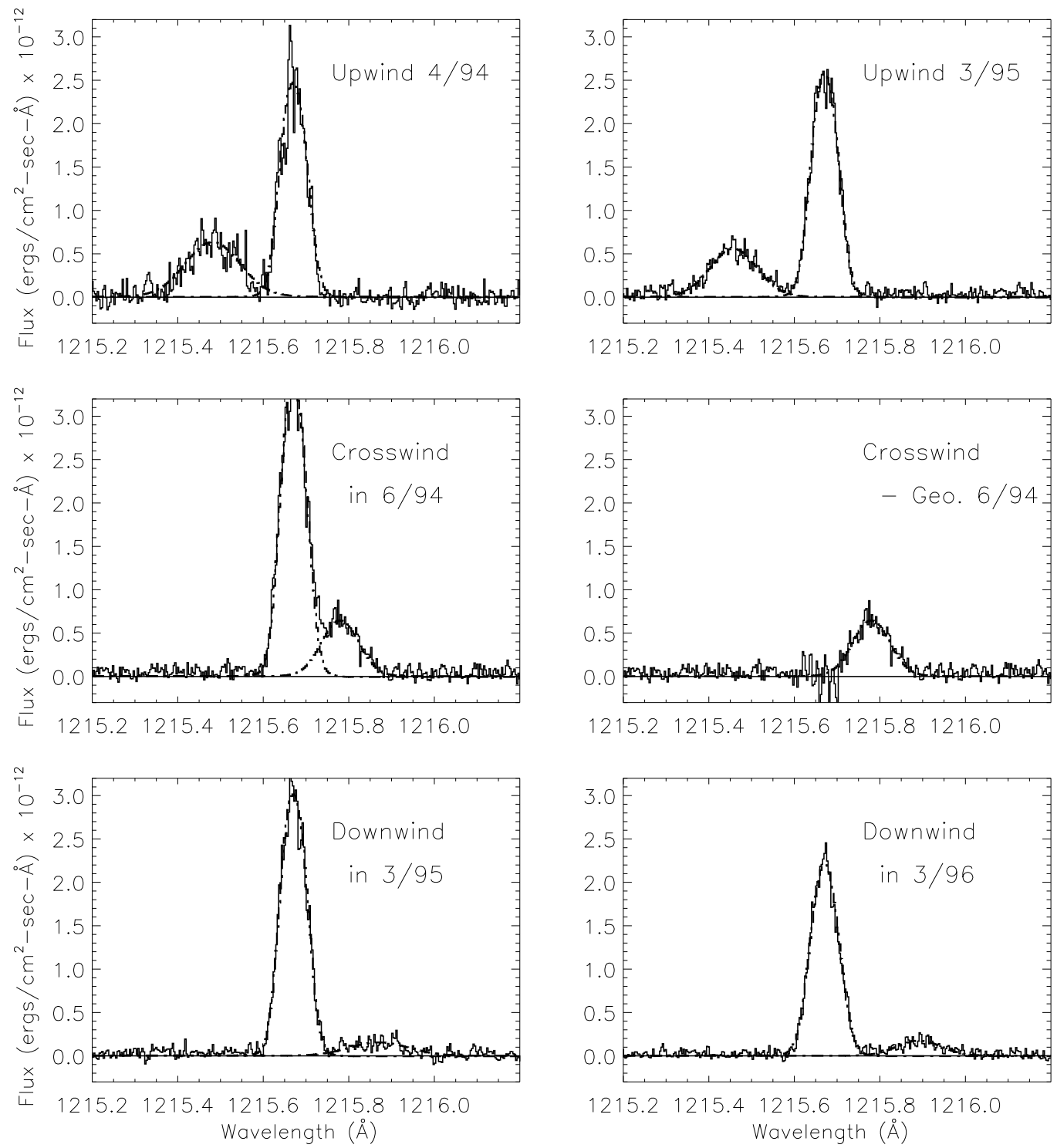

FIG. 2.-Montage of spectra and overplotted fits to geocoronal and IPH emissions for observations over 1994-1996. Fits to the geocoronal and IPH emission lines are Voigt profiles convolved with the GHRS echelle A LSF, and best-fit parameters are listed in Table 2.

do not include variations in solar parameters during the IPH flow through the inner solar system. Since the IPH flow at $20 \mathrm{~km} \mathrm{~s}^{-1}$ is roughly $4 \mathrm{AU}$ per year, variations in solar activity will be reflected in the flow with solar distance, and an improved modeling would include these variations with time.

\subsection{Crosswind}

In the crosswind spectrum, the center of the bright geocoronal line is easily determined, and the magnitude of the model LSF fitted to this line for subtraction then determines the residual shape of the blue wing of the IPH emission. The shape of the IPH line center in the difference spectrum is little affected by reasonable values for the geocoronal subtraction, and the scaling of the geocoronal brightness has been optimized to leave an IPH blue wing symmetric with the observed red wing, within a rather high level of noise in the blue wing from the difference of two large values. With an observation perpendicular to the flow direction, there should be little sensitivity to the assumed inflow speed, and, in fact, assumed values from 10 to 30 $\mathrm{kms}^{-1}$ in the hot-model profiles give reasonable fits. The residual crosswind IPH emission line does appear offset toward the blue of the line center determined solely from the Earth orbital motion, implying a net motion of 2-3 $\mathrm{kms}^{-1}$ of the IPH approaching the Earth along this line of sight, which implies a value of $\mu$ slightly less than 1 . We have also calculated the expected value of $\mu$ for each date of observation (Table 2), taking the measured solar Ly $\alpha$ flux at 1 AU from the Solar Stellar Irradiance Comparison Experiment (SOLSTICE), Tobiska, Pryor, \& Ajello 1997) and then estimating the flux at the position in the line profile appropriate to the solar rest-frame motion of the IPH along the line of sight, using a measured solar line profile (Lemaire et al. 1978). The $\mu$-values based on solar observations are similarly close to or just less than 1 . For the assumed flow direction, values of $\mu$ from 0.8 to 1.0 give good hot-model fits to the observed IPH profile, with good fits also obtained for $T_{D}=(1.0-2.0) \times 10^{6} \mathrm{~s}$. However, the RL and HS models differ from each other by $2-3 \mathrm{kms}^{-1}$ in the IPH line-center 
position for the same assumed parameters, making an exact determination of $\mu$ impossible at this time. An assumed temperature of $8000 \mathrm{~K}$ provides a good fit to the crosswind IPH profile, unlike the upwind and downwind profiles, which appear broader than the model $8000 \mathrm{~K}$ profiles. The possibility of different velocity dispersions in the parallel and perpendicular directions should be considered in future modeling.

\subsection{Downwind}

The IPH parameters are only loosely constrained by the faint downwind IPH line. The assumed values for $\mu$ and $T_{D}$ and inflow speeds of $15-20 \mathrm{kms}^{-1}$ fit the observed profile; but there is little sensitivity to $\mu$ and $T_{D}$, and the inflow speed is constrained only within several $\mathrm{km} \mathrm{s}^{-1}$. It is interesting that the 1996 downwind emission is best fitted with an inflow speed significantly lower than the other observations. However, the low signal-to-noise ratio and greater degree of model sensitivity in the downwind direction require that this finding be verified by other observations. A better fit is obtained for a line broader than the result of a $8000 \mathrm{~K}$ model profile, but with only a weak sensitivity to the exact value of the temperature. Voigt profiles of 15,000 $30,000 \mathrm{~K}$ convolved with the GHRS LSF give plausible fits to the observed emission profiles (Table 2).

\section{DISCUSSION}

The derived parameters for the IPH flow listed in Table 2 are remarkable in the observed variations, both with line of sight and over time in the same viewing direction. The properties of the IPH flow through the inner solar system are clearly more complicated than an assumed uniform flow modified by constant solar parameters. In fitting the flow parameters, the $H S T / G H R S$ crosswind observation constrains the IPH $\mu$-value, and the upwind observations provide determinations of the inflow speed and velocity dispersion. We have compared the observed profiles with the results of two hot models, and compared the results of these models with each other for a range of conditions and lines of sight to determine the sensitivity to the model used. The RL and HS models operate in somewhat different fashions. The RL model calculates the motion and loss rates for each monokinetic parcel of inflowing atoms, then integrates over velocity space and along the line of sight to calculate line profiles. Recent additions to this model, which have been incorporated in this paper, include an estimate of selfabsorption but do not include multiple scattering. The HS model uses the density model of Osterbart \& Fahr 1992, which calculates the $\mathrm{H}$ atom distribution function at each point in space, then integrates along a line of sight and calculates the line profile, including radiative transfer, as described by Scherer \& Fahr (1996). The two hot models yield upwind and downwind line profiles for the same assumed conditions, whose line centers are coincident within $1-2 \mathrm{kms}^{-1}$ with similar line widths (Fig. 3). However, the model profile centroids for the crosswind direction differ by $2-3 \mathrm{~km} \mathrm{~s}^{-1}$, and they display different sensitivities to changing values of $\mu$. A comparison with the model profiles confirms that the $\mathrm{H}$ inflow speed far from the Sun is 18-21 $\mathrm{km} \mathrm{s}^{-1}$, considerably less than the He inflow speed and the speed of the local ISM, and we show that there are variations in the flow speed with line of sight and with time.

Assuming that the measured $\mathrm{He}$ inflow direction is also the $\mathrm{H}$ inflow direction, the observed crosswind blueshift can be reproduced by assuming a value of $\mu$ slightly less than 1 in the hot-model profile, as expected near solar minimum. This observation is the first measurement technique in which the value of $\mu$ can be determined independently of the other parameters. In principle, the observed approaching motion could also be due to an error in the assumed inflow direction, in which case the observed blueshift could be a component of the inflow motion. The inflow direction would need to be approximately $7^{\circ}$ from the assumed direction to reproduce the observed blueshift, which is comparable to the uncertainty in the determination of the Ly $\alpha$ intensity maximum (Ajello et al. 1987). Future crosswind observations both toward and opposite the Earth orbital motion could address the directional uncertainty, since a net focusing of the IPH near the Sun $(\mu<1)$ would produce a blueshift of the IPH line in both directions. Observations with higher spectral resolution (to separate the IPH from the geocorona) would also allow crosswind measurements along lines of sight above and below the ecliptic plane, corresponding to a range of solar latitudes and radiation pressures, to test the extent to which $\mu$ varies with solar latitude and activity. These measurements will be needed to derive an accurate picture of the three-dimensional IPH flow through the solar system.

The difference in velocity distributions between the upwind/downwind and crosswind profiles is a significant development in our understanding of the near-solar flow region. Earlier derivations of the IPH temperature gave values near $8000 \mathrm{~K}$, with these observations limited to regions of the sky $\pm 45^{\circ}$ of the crosswind direction because of the limited spectral range of absorption cells. The HST/ GHRS observations are consistent with these line widths along a similar line-of-sight crosswind, but at the same time indicate much greater velocity widths both upwind and downwind. We interpret this as reflecting an increase in the velocity dispersion in the flow direction from a combination of solar attraction/repulsion, plus any modification by charge-exchange reactions in the heliospheric interface region. In this case the derived temperatures listed in Table 2 are effective fits to the profiles, while the actual velocity distributions are likely to be nonthermal in nature. These velocity distributions could be derived more accurately from the observed line profiles with higher signal-to-noise data at a similar or slightly higher spectral resolution. Note that the present derivation of flow parameters does not use the absolute intensities, which have been measured and are listed in Table 1. The intensities that we have observed are similar to earlier quoted observations near solar minimum (Wu \& Judge 1980; Bertaux et al. 1985; Ajello et al. 1987; Pryor et al. 1992). The modeled intensities can be made to fit the observed profiles by varying either the IPH density or the solar flux, and the density does not affect the velocity distribution while the solar flux has been determined through the limits on $\mu$ from the crosswind profile.

While the increased velocity dispersion in the flow direction may be consistent with either a variable solar flux or modification at the heliospheric interface, the overall decrease in flow speed for $\mathrm{H}$ atoms within the solar system appears to require a substantial shift in the velocity distribution at large distances from the Sun. The inflow direction would have to differ from the assumed line of sight by more than $30^{\circ}$ to explain a $5-8 \mathrm{kms}^{-1}$ velocity decrease by an alteration of the flow direction from that for $\mathrm{He}$ atoms, which would be inconsistent both with the direction of the 

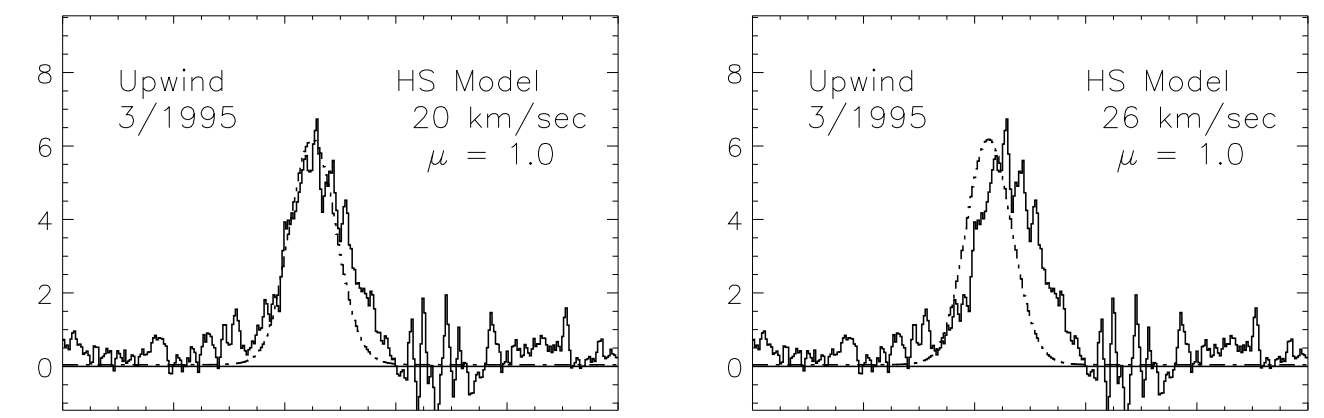

$1215.0 \quad 1215.2 \quad 1215.4 \quad 1215.6 \quad 1215.8 \quad 1216.0$

$1215.0 \quad 1215.21215 .4 \quad 1215.6 \quad 1215.8 \quad 1216.0$
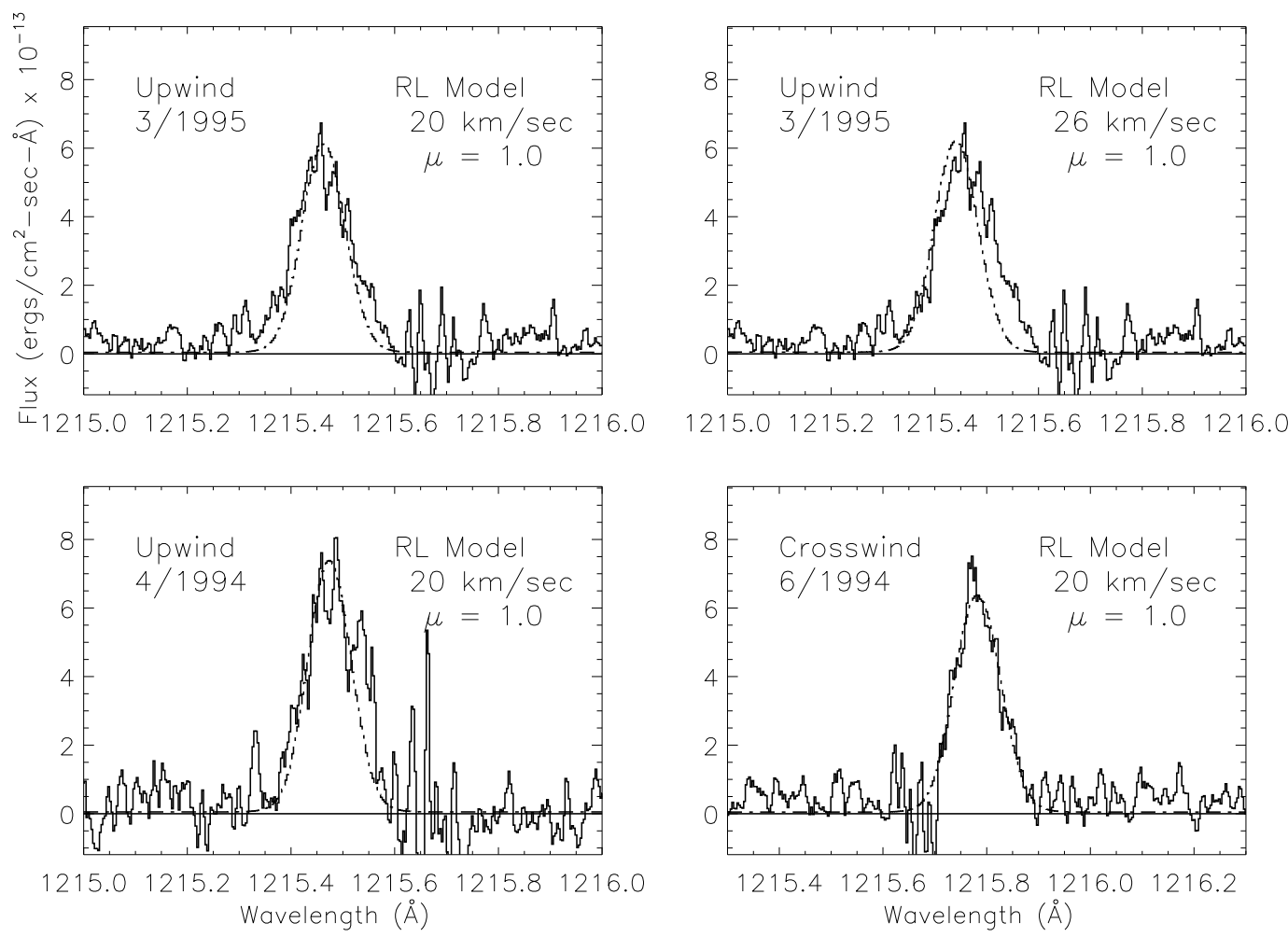

FIG. 3.-Comparison of hot-model profiles with observed GHRS IPH data for selected cases and lines of sight, demonstrating the sensitivity to assumed inflow speed and hot model used.

observed $\mathrm{H}$ intensity maximum (Ajello et al. 1987) and with prior absorption cell measurements (Bertaux et al. 1985). The added observations of different parallel and perpendicular velocity distributions, and different flow speed and IPH temperatures at different times and along different lines of sight, appear consistent with some results of recent modeling by Williams et al. (1997). The inclusion of Hproton and $\mathrm{H}-\mathrm{H}$ thermalizing collisions, in addition to charge-exchange reactions between solar wind protons and IPH neutrals, led these authors to suggest that the IPH H flow should have an increased velocity dispersion as well as an overall decrease in flow speed. These and other interpretations of the line profiles will benefit from further comparison with the observational data.
We acknowledge assistance from the Space Telescope Science Institute in the scheduling and reduction of the HST observations. This research was supported by grant GO-4604.01-92A from the Space Telescope Science Institute to the University of Michigan. H. F. and H. S. were supported by a grant " $H S T$-Rutolgman" from the German Space Research Foundation DARA. This work is based on observations with the NASA/ESA Hubble Space Telescope, obtained at the Space Telescope Science Institute, which is operated by AURA, Inc., for NASA under contract NAS 5-26555.

\section{REFERENCES}

Adams, T. F., \& Frisch, P. 1977, ApJ, 212, 300

Ajello, J. M., Pryor, W. R., Barth, C. A., Hord, C. W., Stewart, A. I. F., Simmons, K. E., \& Hall, D. T. 1994, A\&A, 289, 283

Ajello, J. M., et al. 1987, ApJ, 317, 964

Baranov, V. B., Lebedev, M. G., \& Malama, J. G. 1991, ApJ, 375, 347
Bertaux, J. L., \& Blamont, J. E. 1971, A\&A, 11, 200

Bertaux, J. L., Blamont, J. E, Tabarle, N., Kurt, V. G., Bourgin, M. C., Smirnov, A. S., \& Denenteva, N. N. 1976, A\&A, 46, 19

Bertaux, J. L., Lallement, R., Kurt, V. G., \& Mironova, E. N. 1985, A\&A, 150,1 
Clarke, J. T., Bowyer, S., Fahr, H., \& Lay, G. 1984, A\&A, 139, 389

Clarke, J. T., Lallement, R., Bertaux, J. L., \& Quemerais, E. 1995, ApJ, 448, 893

Fahr, H. J., \& Ripken, H. W. 1984, A\&A, 139, 551

Hall, D. T., et al. 1993, J. Geophys. Res., 98, 15185

Lallement, R., Bertaux, J. L., \& Clarke, J. T. 1993, Science, 260, 1095

Lallement, R., Bertaux, J. L., \& Dalaudier, F. 1985, A\&A, 150, 21

Lallement, R., \& Bertin, P. 1992, A\&A, 266, 479

Lemaire, P., et al. 1978, ApJ, 223, L55

Linsky, J. L., et al. 1993, ApJ, 402, 694

Malama, J. G. 1991, Ap\&SS, 176, 21

Osterbart, R., \& Fahr, H. J. 1992, A\&A, 264, 260

Pryor, W. R., et al. 1992, ApJ, 394, 363
Puyoo, O., BenJaffel, L., \& Emerich, C., 1997, ApJ, 480, 262

Quemerais, E., Lallement, R. L., \& Bertaux, J. L. 1993, A\&A, 277, 283

Ripken, H. W., \& Fahr, H. J. 1983, A\&A, 122, 18

Scherer, H., \& Fahr, H. J. 1996, A\&A, 309, 957

Scherer, H., Fahr, H. J., \& Clarke, J. T., 1997, A\&A, 325, 745

Thomas, G. E., \& Krassa, R. F. 1971, A\&A, 11, 218

Tobiska, W. K., Pryor, W. R., \& Ajello, J. M. 1997, Geophys. Res. Lett., 24, 1123

Weller, C. S., \& Meier, R. R. 1974, ApJ, 193, 471

Williams, L. I., Hall, D. T., Pauls, H. L., \& Zank, G. P. 1997, ApJ, 476, 366

Witte, M., et al. 1993, Adv. Space Res., 13(6), 121

Wu, F. M., \& Judge, D. L. 1980, ApJ, 239, 389 